\title{
NEW AND RARE SPIDER SPECIES (ARACHNIDA, ARANEAE) FROM UKRAINE
}

\author{
N. Polchaninova ${ }^{1^{*}}$, V. Gnelitsa ${ }^{2}$, V. Terekhova ${ }^{1}$, A. Iosypchuk ${ }^{1,3}$ \\ ${ }^{1}$ V. N. Karazin Kharkiv National University, Svobody sq., 4, Kharkiv, 61022 Ukraine \\ ${ }^{\star}$ E-mailn.polchaninova@karazin.ua \\ E-mailv.terekhova@karazin.ua \\ ${ }^{2}$ Sumy State University, Rimsky-Korsakov st., 2, Sumy, 40000 Ukraine \\ E-mailgnelitsav@gmail.com \\ ${ }^{3}$ Kherson State University, University st., 27, Kherson, 73003 Ukraine \\ E-mailiosipchuk.nastya@ukr.net \\ ${ }^{*}$ Corresponding author \\ N. Polchaninova (https://orcid.org/0000-0003-4605-8788) \\ V. Gnelitsa (https://orcid.org/0000-0003-1833-4386) \\ V. Terekhova (https://orcid.org/0000-0002-6655-9072) \\ A. Iosypchuk (https://orcid.org/0000-0002-8242-002X)
}

New and Rare Spider Species (Arachnida, Araneae) from Ukraine. Polchaninova, N., Gnelitsa, V., Terekhova, V., Iosypchuk, A. - An annotated list of 31 spider species of 12 families collected in Northeasten, Easten and Southern Ukraine is presented. Four speceis, Marinarozelotes manychensis (Ponomarev \& Tsvetkov, 2006), Turkozelotes kazachstanicus (Ponomarev et Tsvetkov, 2006), Zelotes atrocaeruleus (Simon, 1878), and Z. puritanus Chamberlin, 1922, are recorded for the first time from Ukraine. For Micaria coarctata (Lucas, 1846), this is the first record from the East European Plain. Nine species were recorded for the first time from Left-Bank Ukraine. To date, 1081 spider species are known from Ukraine and 757 species from Left-Bank Ukraine. Based on recent data, the northern boundaries of the geographic ranges of Drassyllus crimeaensis Kovblyuk, 2003 and Bassanioides caperatus (Simon, 1875) run along the northern coast of the Sea of Azov. For Marinarozelotes manychensis and Turkozelotes kazachstanicus the northwestern coast of the Sea of Azov is the westernmost known limit of their distiribution. The range of Heriaeus horridus Tyschchenko, 1965 is delimited in the west by the right bank of the Dnipro estuary. Thirteen species are illlustrted.

Key words: araneofauna of Europe, geographic distribution, habitat preference, conservation areas. 


\section{Introduction}

Arachnological research in Ukraine has a long history dating back to the 1830s (Polchaninova \& Prokopenko, 2019). Until now, 1077 spider species have been known in the Ukrainian fauna (Nentwig et al., 2021). Nonetheless, the country's territory is still unevenly studied. The Crimea and Left-Bank Ukraine are investigated best. Spiders of these regions have been catalogued, and the species lists are constantly updated. A list of spiders of the Crimea numbers 569 species (Kovblyuk \& Kastrygina, 2015; Nadolny, 2020), and that of Left-Bank Ukraine 741 species (Polchaninva \& Prokopenko, 2013, 2017, 2019). Even in these well-studied areas, there are many blank spots. One of them is Zaporizhzhia Region, with only 122 species known. For comparison, a list of spiders of the adjacent Donetsk Region numbers 514 species (Polchaninva \& Prokopenko, 2019).

The aim of the present paper is to make a list of recently recorded and rare spider species in Ukraine, to comment on their geographical distribution, and to provide photos/drawings of the species, which need additional illustration.

\section{Material and methods}

The material was collected during field expeditions by N. Polchaninova and diploma studies by R. Honcharov (V. N. Karazin Kharkiv National University) in May-October 2019, and collecting trips by A. Iosipchuk (Kherson State University) in 2020 in 23 localities in Donetsk, Kharkiv, Kherson and Zaporizhzhia administrative regions of Ukraine. Hereinafter, we use the abbreviations NNP - National Nature Park, RLP Regional Landscape Park, AR Crimea - Autonomic Republic of Crimea, Reg. - administrative Region. Unless otherwise specified, the material was collected by N. Polchaninova; pitfall trapping was the main sampling method.

List of collecting localities mentioned in the text.

- $800 \mathrm{~m}$ E Bohorodychne [Богородичне], NNP 'Sviati Hory', $49.02111^{\circ} \mathrm{N}, 37.51972^{\circ} \mathrm{E}$.

- $\quad 1.7 \mathrm{~km}$ E Davydivka [Давидівка], Utliutskyi Lyman, the Mala River mouth, Pryazovskyi NNP, $46.51200^{\circ} \mathrm{N}$, $35.18170^{\circ} \mathrm{E}$.

- $\quad 2 \mathrm{~km}$ E Davydivka [Давидівка], Pryazovskyi NNP, $46.51509^{\circ} \mathrm{N}, 35.18784^{\circ} \mathrm{E}$.

- $\quad 2.7 \mathrm{~km} \mathrm{~S}$ Dvorichna [Дворічна], $49.82833^{\circ} \mathrm{N}, 37.67583^{\circ} \mathrm{E}$.

- $4 \mathrm{~km}$ E Krasnokutsk [Краснокутськ], NNP 'Slobozhanskyi', $50.06167^{\circ} \mathrm{N}, 35.22583^{\circ} \mathrm{E}$.

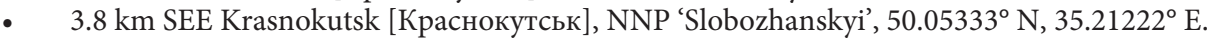

- $2 \mathrm{~km}$ SW Kryva Luka [Крива Лука], Department 'Kreidova Flora' of the Ukrainian Natural Steppe Reserve, $48.86944^{\circ} \mathrm{N}, 37.87306^{\circ} \mathrm{E}$.

- $\quad 800$ m SW Kryva Luka [Крива Лука], 'Kreidova Flora', $48.87444^{\circ}$ N, $37.88972^{\circ}$ E.

- $\quad 1.3 \mathrm{~km}$ NE Kryva Luka [Крива Лука], 'Kreidova Flora', $48.87861^{\circ} \mathrm{N}, 37.91889^{\circ} \mathrm{E}$.

- $\quad 2$ km NE Kryva Luka [Крива Лука], 'Kreidova Flora', $485249^{\circ}$ N, $375545^{\circ} \mathrm{E}$.

- $\quad$ Nesterivka [Нестерівка], RLP 'Velykoburlutskyi Steppe’, $49.93028^{\circ} \mathrm{N}, 37.30944^{\circ} \mathrm{E}$

- $\quad 1.5 \mathrm{~km}$ NEE Nove [Нове], Utliutskyi Lyman, the Atmanai River mouth, Pryazovskyi NNP, $46.44021^{\circ} \mathrm{N}$, $35.10316^{\circ} \mathrm{E}$.

- $\quad 1.3 \mathrm{~km}$ E Nove [Нове], Pryazovskyi NNP, $46.43720^{\circ} \mathrm{N}, 35.09892^{\circ} \mathrm{E}$

- $\quad 1.2 \mathrm{~km}$ NNE Respublikanets [Республіканець], NNP 'Kamyanska Sich', $47.01500^{\circ}$ N, $33.658056^{\circ} \mathrm{E}$.

- $1 \mathrm{~km}$ NW Respublikanets [Республіканець], NNP 'Kamyanska Sich', $47.00833^{\circ} \mathrm{N}, 33.64028^{\circ} \mathrm{E}$.

- $500 \mathrm{~m}$ SSE Respublikanets [Республіканець], NNP 'Kamyanska Sich’, $46.99972^{\circ} \mathrm{N}, 33.65361^{\circ} \mathrm{E}$.

- $\quad 4.8 \mathrm{~km}$ NE Sheliuhy [Шелюги], Molochnyi Lyman coast, Pryazovskyi NNP, $46.59287^{\circ} \mathrm{N}, 35.27463^{\circ} \mathrm{E}$.

- $\quad 1.85 \mathrm{~km}$ SW Shyroka Balka [Широка Балка], Dniprovskyi Lyman coast, $46.57629^{\circ} \mathrm{N}, 32.18361^{\circ} \mathrm{E}$.

- $\quad 3.5 \mathrm{~km}$ SW Stepok [Степок], Fedotova spit, Pryazovskyi NNP, $46.27172^{\circ} \mathrm{N}, 35.28255^{\circ} \mathrm{E}$.

- $\quad 5 \mathrm{~km}$ W Stepanivka [Степанівка], Stepanivska spit, Pryazovskyi NNP, $46.43719^{\circ} \mathrm{N}, 35.44617^{\circ} \mathrm{E}$.

- Syvashyk bay shore, Utliutskyi Lyman [берег затоки Сивашик Утлюцького лиману] Pryazovskyi NNP, $46.41398^{\circ} \mathrm{N}, 35.10038^{\circ} \mathrm{E}$.

- $\quad 3.9 \mathrm{~km}$ NNW Novokairy [Новокаїри], NNP 'Kamyanska Sich', $46.99972^{\circ} \mathrm{N}, 33.65361^{\circ} \mathrm{E}$.

- $\quad 3.9 \mathrm{~km}$ W Skadovsk [Скадовськ], NNP 'Dzharylhatskyi', $46.12084^{\circ} \mathrm{N}, 32.86619^{\circ} \mathrm{E}$.

We listed here only remarkable records referred to the spider species: first records from Ukraine marked with an asterisk $\left({ }^{*}\right)$, first records from Left-Bank Ukraine, and patchily distiributed rare species.

The nomenclature follows the World Spider Catalog (WOS, 2021). A list of species is provided with a name of locality (see above), habitat, number of males/females, and collecting date(s) as well as data on the species distribution in Ukraine and general disitribution. The species ranges are given after the catalogues of regional and world faunas (Mikhailov, 2013, 2021; Kovblyuk \& Kastrygina, 2015; Li \& Lin, 2016; Polchaninova \& Prokopenko, 2013, 2017, 2019; Nentwig et al., 2021; WOS, 2021). In case of the absence of generalizing works or for the range specification, we use appropriate papers (see below). The material is deposited in N. Polchaninova's personal collection (Kharkiv, Ukraine). 
We have chosen 13 species for illustration based on the following criteria: the species copulatory organs are drawn exhaustively but there are no photos; the species is illustrated with photos, but the more detailed drawings are needed; the species general appearance is not illustrated well. Moreover, some species were found at the extremities of their ranges, thus, their morphological peculiarities can demonstrate the species geographic variability. The photos were taken by V. Terekhova with the use of the camera Leica DC 300 and the binocular microscop Leica MZ 7.5, the ink drawings were made by V. Gnelitsa with a camera lucida.

\section{List of species}

\section{Family Clubionidae \\ Porrhoclubiona genevensis (L. Koch, 1866)}

Material examined. Kharkiv Reg., near Nesterivka, SE facing slope dominated by grasses 28.05-30.06.2019, 1 o'.

Distribution. Europe, Canary Islands, North Africa, Central Asia (to Tajikistan) (Zamani et al., 2017; Marusik \& Omelko, 2018). Ukraine: Kyiv Reg.: Middle Dnipro region (Singaevsky, 2014 as Clubiona g.), Kopachiv (E. Singaevsky pers. comm); AR Crimea: Saky Distr. (Kovblyuk \& Kastrygina, 2015 as Clubiona g.); Kharkiv Reg. (first record).

Note. First record from Left-Bank Ukraine.

\section{Family Dictynidae}

Devade tenella (Tystshenko, 1965) (fig. 1)

Material examined. Zaporizhzhia Reg., 4 km E Sheliuhy, saline marshes, 07-24.06.2019, 5 o; 2 km E Davydivka, saline marshes, 07-24.06.2019, 6 ○’.

Distribution. From Southern Ukraine (Kovblyuk \& Kastrygina, 2015; Polchaninova \& Prokpenko, 2019) to China (Xinjiang) (Li \& Lin, 2016). Ukraine: Kherson Reg.: Heroiske, Novochornomorya; Donetsk Reg.: Donetsk, Siedove (Polchaninova \& Prokopenko 2013, 2017); AR Crimea: Simferopol Distr. (Kovblyuk \& Kastrygina 2015); Zaporizhzhia Reg. (first record).

Note. A saline marshes specialist. Heroiske Vill. (Kherson Reg.) is the westernmost known locality.

\section{Dictyna sinuata Esyunin \& Sozontov, 2016 (figs 2-5)}

Material examined. Kharkiv Reg., 1.7 km S Dvorichna, foot of a chalk hill, 17.05.2019, 1 o'.

Distribution. Only three localities are known. Russia: Orenburg Reg. (Esyunin \& Sozontov, 2016). Ukraine: Kherson (Ponomarev et al., 2017 c) and Kharkiv (first record) regions.

Note. First record from Left-Bank Ukraine.

\section{Family Gnaphosidae Callilepis schuszteri (Herman, 1879)}

Material examined. Kharkiv Reg., 4 km E Krasnokutsk, young pine plantation, 23.05.-16.06.2019, 10 ơ, 2 o, (R. Honcharov); idem, bank of a bog, 23.05-6.06.2019, 12 ơ, 1 ○ (R. Honcharov); $3.8 \mathrm{~km} \mathrm{SEE}$ Krasnokutsk, old pine forest, 16.06-4.07.2019, 4 O' (R. Honcharov).

Distribution. Widespread in Europe (Nentwig et al., 2021). Ukraine: Kyivske Polissia (Evtushenko, 1991), Kharkiv Reg. (first record). Further to the east occurs from West Siberia (Kemerovo) (Trilikauskas, 2013) to the Russian Far East (Mikhailov, 2013; WOS, 2021).

Note. First record from the Wood-and-Steppe zone of Ukraine and the easternmost known locality in the East European Plain. 


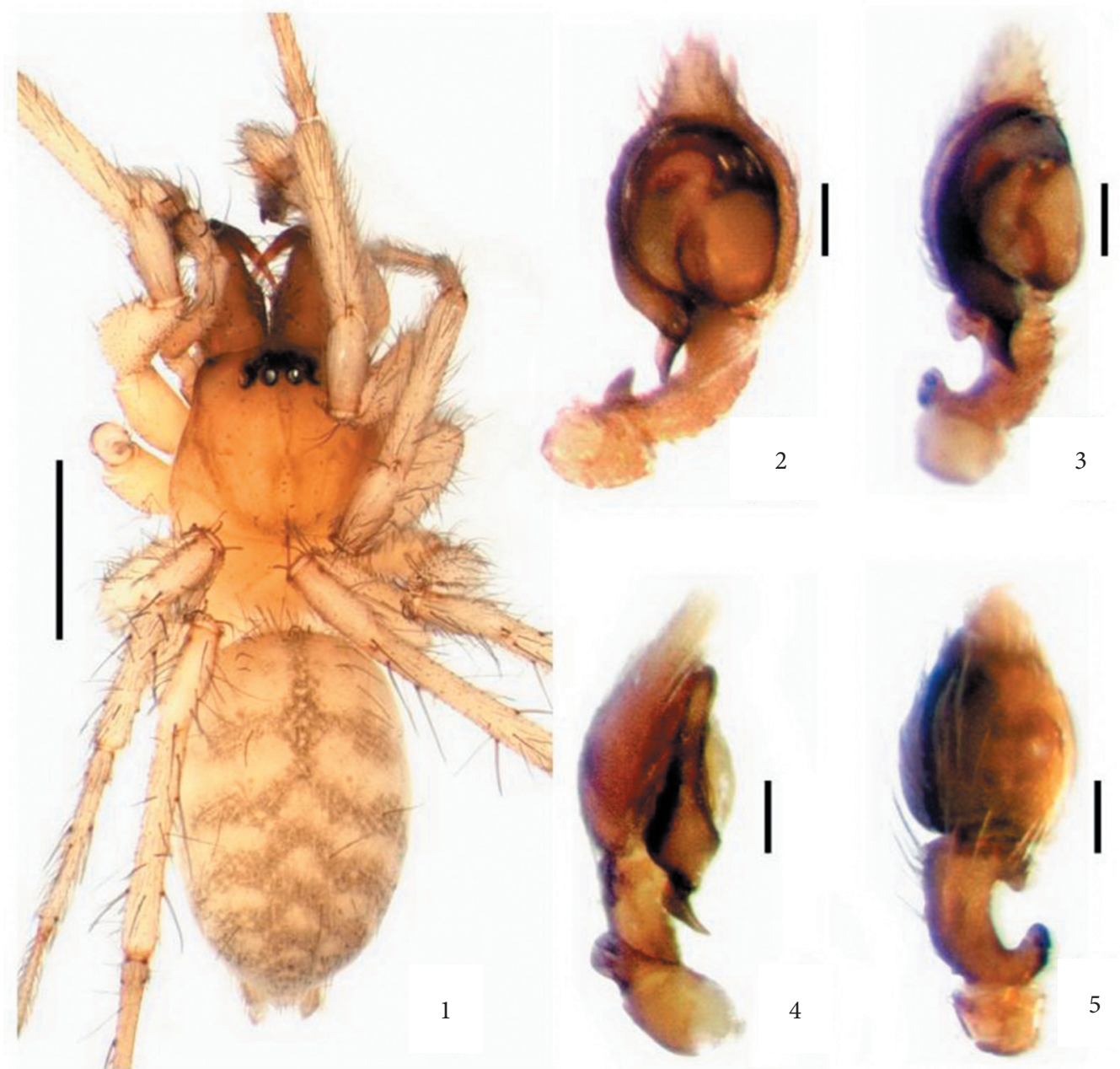

Figs 1-5. Devade tenella: 1 - general appearance dorsal; Dictyna sinuata: male palp: 2 - ventral, 3 - ventralprolateral, 4 - prolateral, 5 - dorsal. Scale bars: fig. $1-1 \mathrm{~mm}$; figs. $2-5-0.1 \mathrm{~mm}$.

Drassyllus crimeaensis Kovblyuk, 2003 (fig. 6)

Material examined. Zaporizhzhia Reg., 1.7 km E Davydivka, sandy steppe, 07-24.06.2019, 1 ; $4.8 \mathrm{~km}$ NE Sheliuhy, steppe slope on the lyman coast, 07-24.06.2019, 1 ○; Syvashyk, saline marshes, 10-22.06.2019, 10 ○', 1 . .

Distribution. North Macedonia, Greece, Turkey (European and Asian parts) (Nentwig et al., 2021); Russia (Rostov-on-Don Reg., Krasnodar Krai, Dagestan) (Ponomarev, 2008; Ponomarev et al., 2014, 2019); Azerbaijan (Mikhailov, 2013). Ukraine: AR Crimea: Lenino, Saky, Simferopol, Feodosiya, Yalta distr., Kerch (Kovblyuk \& Kastrygina, 2015); Zaporizhzhia Reg. (first record).

Note. A record "Yalta, Donetsk Area" in Polchaninova \& Prokopenko (2013) was excluded from the list of collecting localities due to loss of the material (Polchaninova \& Prokopenko, 2019). Hence, the present data are the first valid records from Left-Bank Ukraine.

Gnaphosa ukrainica Ovtsharenko, Platnick \& Song, 1992

Material examined. Kherson Reg., 500 m SSE Respublikanets, medium-height riverbank dominated by forbs, 15.06-3.07.2020, 1 o (Iosypchuk); 3.9 km W Skadovsk, salt marshes, 24.07.-01.08.2019, 1 o (Iosypchuk); 
Zaporizhzhia Reg., 2 km E Davydivka, saline marshes, 07-24.06.2019, 32 ơ, 11 ९; 4.8 km NE Sheliuhy, saline marshes, 07-24.06.2019, 31 ○', 4 ㅇ.

Distribution. From Southern Ukraine to West Siberia (Novosibirsk Reg., Russia) (Azarkina et al., 2018), southward to Turkmenistan (Mikhailov, 2013) and Iran (Kerman Province) (Zamani \& Marusik, 2018). Ukraine: Kherson Reg.: Heroiske, Novochornomorya (Polchaninova \& Prokopenko, 2013), Respublikanets (Iosypchuk, 2019), Skadowsk (Iosypchuk et al., 2020); AR Crimea: Saky Distr. (Kovblyuk \& Kastrygina, 2015); Zaporizhzhia Reg. (first record).

Note. Heroiske Vill. (Kherson Reg.) is the westernmost known locality. Being abundant on saline marshes, the species has a highly patched distribution: viz., in the Pryazovslyi NNP, it was found in two localities out of five, although all of them host vast saline marshes.

\section{Marinarozelotes manychensis (Ponomarev \& Tsvetkov, 2006)*}

Material examined. Zaporizhzhia Reg., 1.5 km NNE Nove, saline marshes, 10-22.06.2019, 1 ơ, 1 \&.

Distribution. Russia: Rostov-on-Don Reg., Stavropol Krai, Dagestan, Orenburg Reg. (Esyunin \& Tuneva, 2020 (as Trachyzelotes m.); Ponomarev \& Shmatko, 2020). Ukraine: Zaporizhzia Reg. (first record).

Note. New to Ukraine. Nove Vill. is the westernmost known locality.

\section{Micaria bosmansi Kovblyuk \& Nadolny, 2008}

Material examined. Kherson Reg., $1 \mathrm{~km}$. NW Respublikanets, secondary steppe in a shallow gully, 23.05-22.06.2019, 1 o (Polchaninova \& Iosypchuk); 500 m SSE Respublikanets, medium-height riverbank dominated by forbs, 15.06-3.07.2020, 1 ○' (Iosypchuk); shrub thickets on the medium-height riverbank,

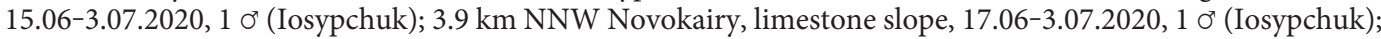
Zaporizhzhia Reg., 4.8 km NE Sheliuhy, steppe slope on the lyman coast, 07-24.06.2019, 1 o.

Distribution. From Ukraine (Iosypchuk 2019; Polchaninova et al., 2021) and Niğde Province of Turkey (Demir et al., 2015) to the Orenburg Reg. of Russia (Esyunin \& Tuneva, 2020). Ukraine: Dnipropetrovsk Reg.: Kryvyi Rih (Polchaninova et al., 2021); Luhansk Reg.: Striltsivka, Triokhizbenka (Polchaninova \& Prokpenko, 2013); Kherson Reg.: Respublikanets (Iosypchuk, 2019); AR Crimea: Sevastopol, Feodosiya, Yalta distr.; Tarkhankut peninsula (Kovblyuk \& Kastrygina, 2015; Nadolny, 2020); Zaporizhzhia Reg. (first record).

Note. Novokairy Vill. (Kherson Reg.) and Kryvyi Rih Town (Dnipropetrovsk Reg.) are the westernmost known localities.

\section{Micaria coarctata (Lucas, 1846) (figs 8, 13-14)}

Material examined. Zaporizhzhia Reg., 1.7 km E Davydivka, sandy steppe, 07-24.06.2019, 3 ơ.

Distribution. From Southwestern Europe and Northwestern Africa to the Russian Far East (Mikhailov, 2013; Nentwig et al., 2021; WOS, 2021). Ukraine: AR Crimea: Saky, Feodosiya distr.; Tarkhankut peninsula (Kovblyk \& Kastrygina, 2015; Nadolny, 2020); Zaporizhzhia Reg. (first record).

Note. First record from the East European Plain, first record from Left-Bank Ukraine. The collecting locality lies on the northern boundary of the species range.

\section{Talanites strandi Spassky, 1940}

Material examined. Zaporizhzhia Reg., 1.7 E Davydivka, sandy steppe, 07-24.06.2019, 3 ơ; 1.3 km E Nove, abandoned field, 10-22.06.2019, 1 O'; 5 km W Stepanivka, mesic saline meadow, 8-23.06.2019, 1 o'. 
Distribution. From Southern Ukraine (Kovblyk \& Kastrygina, 2015) to Western Kazakhstan (Piterkina \& Ovtcharenko, 2009). Ukraine: AR Crimea: Sudak, Feodosiya distr.; Tarkhankut peninsula (Kovblyk \& Kastrygina, 2015; Nadolny, 2020); Donetsk Reg.: Amvrosievka (Spassky, 1940); Zaporizhzhia Reg. (first record). in 1940.

Note. This is the second record from mainland Ukraine since the species description

Turkozelotes kazachstanicus (Ponomarev et Tsvetkov, 2006)* (figs. 9-10)

Material examined. Zaporizhzhia Reg., Syvashyk, saline marshes, 10-22.06.2019, 1 o', 1 o.

Distribution. Russia: Rostov-on-Don Reg. (Ponomarev \& Dvadnenko, 2011 as T. mirandus). Kazakhstan: Atyrau and Kostanay reg. Azerbaijan: Nakhchivan AR (Ponomarev et al., 2021). Iran: Ilam Province (Zamani et al., 2018 as T. mirandus). Ukraine (first record): Zaporizhzhia Reg. (first record). locality.

Note.A saline marshes specialist. Syvashyk bay shore is the westernmost known

\section{Zelotes atrocaeruleus (Simon, 1878)*}

Material examined. Zaporizhzhia Reg., Syvashyk, saline marshes, 10.05.-06 June 2019, 1 o'.

Distribution. From France and Spain (Nentwig et al., 2021) to Northeastern Kazakhstan (Mikhailov, 2013) and Xinjiang Province of China ((Li \& Lin, 2016). Ukraine (first record): Zaporizhzhia Reg. (first record).

Zelotes erebeus (Thorell, 1871) (fig. 7)

Material examined. Donetsk Reg., $800 \mathrm{~m}$ E Bohorodychne, open pine forest on a chalk hill, 09.09.-03.10.2019, 6 ơ.

Distribution. The species is distributed in Central and Western Europe (Rozwałka et al., 2016; Nentwig et al., 2021). In the East European Plain, it is known from several localities detached from the main range. Russia: Mari-El Republic (Matveev et al., 2003), Samara Reg. (Krasnobayev, 2007). Ukraine: Zakarpattia Reg.: Mala Uholka (Prokopenko et al., 2014); Cherkassy Reg.: (as Middle Dnipro region in Singaevsky, 2014); Donetsk Reg. (first record).

Note. First record from Left-Bank Ukraine.

Zelotes prishutovae Ponomarev \& Tsvetkov, 2006 (figs 11-12)

Material examined. Zaporizhzhia Reg., Syvashyk, saline marshes, 10-22.06.2019, 1 o; $5 \mathrm{~km}$ W Stepanivka, mesic saline meadow, 10-22.06.2019, 1 o'.

Distribution. Greece (Senglet, 2011); South of European Russia: Rostov-on-Don and Volgograd reg., Stavropol Province (Ponomarev \& Khnykin, 2013; Ponomarev, 2017; Ponomarev et al., 2017 a, b). Ukraine: AR Crimea: Feodosiya (Kovblyuk et al., 2009 as Camillina metellus (Roewer, 1928). According to Senglet (2011), the Crimean individual belongs to Z. prishutovae; Zaporizzhia Reg. (first record).

Note. The northern boundary of the species range runs along the northern coast of the Sea of Azov. First record from Left-Bank Ukraine.

Zelotes puritanus Chamberlin, 1922*

Material examined. Kharkiv Reg., 4 km E Krasnokutsk, young pine plantation, 23.05.-16.06.2019, 2 ơ, 1 ○ (R. Honcharov); idem, bank of a bog, 23.05.-16.06.2019, 1 @ (R. Honcharov); idem, 16.06.-4.07.2019, 1 ơ, 10 @ (R. Honcharov). 
Distribution. Circumholarctic disjunctive range (WSC, 2020). Ukraine (first record): Kharkiv Reg.

\section{Zelotes pseudogallicus Ponomarev, 2007 (figs 15-18)}

Material examined. Donetsk Reg., $2 \mathrm{~km} \mathrm{SW}$ Kryva Luka, bottom of a shallow gully with trees, 06.05.-02.06.2019, 1 ơ; $2 \mathrm{~km}$ NE Kryva Luka, top of a chalk hill with sparse vegetation, 06.05.-02.06.2019, 1 \%; idem, edge of the Caragana thickets on a chalk hill, 06.05.-02.06.2019, 1 \%.

Distribution. From Eastern Ukraine (Polchaninova \& Prokopenko, 2017) to West Siberia (Tobolsk Reg., Russia; Kostanay Reg., Kazakhstan) (Ponomarev \& Shmatko, 2019). Ukraine: Lugansk Reg.: Krynychne, Shchastia (Polchaninova \& Prokopenko, 2013, 2017); Donetsk Reg.: Sviatohirsk (Polchaninova \& Prokopenko, 2017).

Note. Kryva Luka Vill. and Sviatohirsk Town are the westernmost known localities.

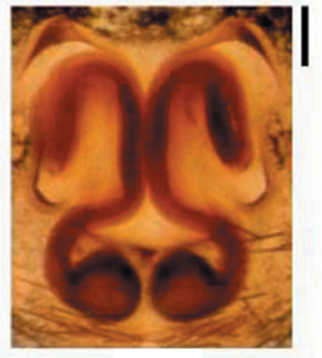

6

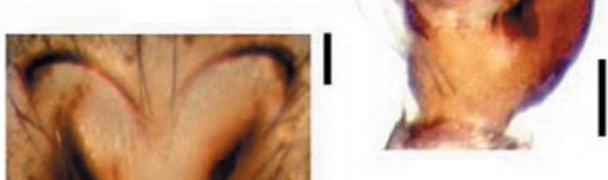

9

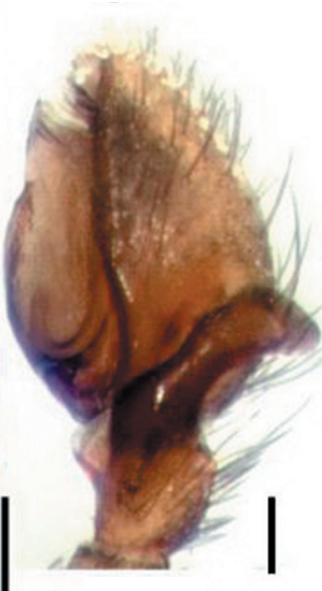

10

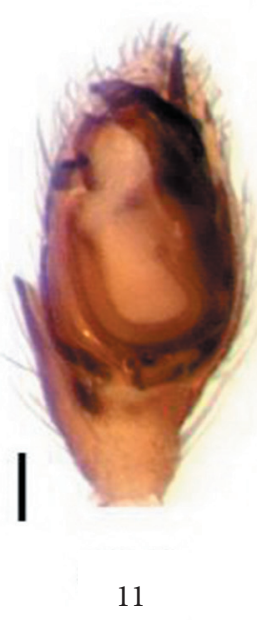

11

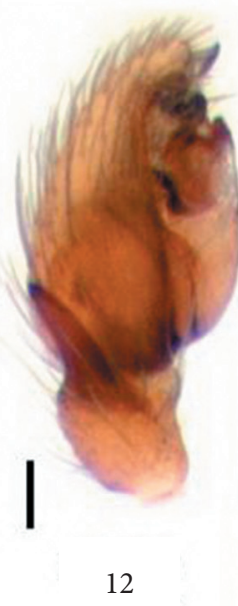

12

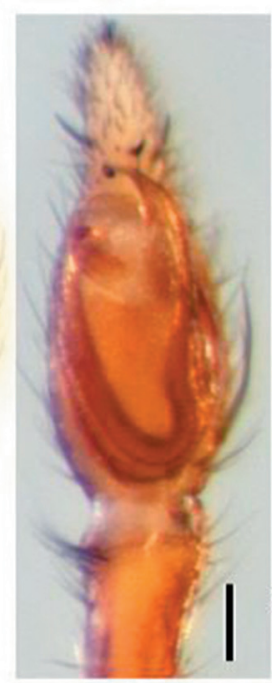

13

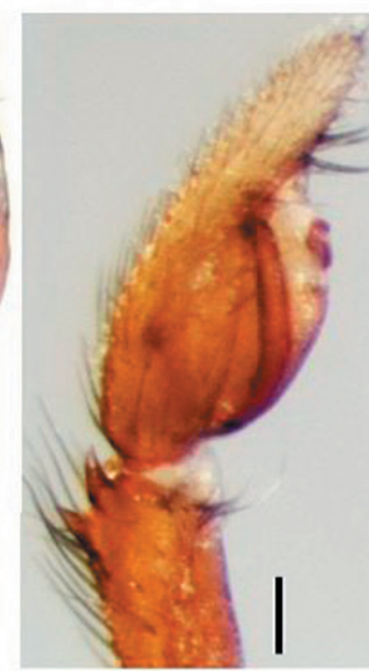

14

8

Figs 6-14. Drassyllus crimeaensis: 6 - epigyne ventral; Zelotes erebeus: 7 - epigyne ventral; Micaria coarctata: 8 - carapace dorsal, male palp: 13 - ventral, 14 - retrolateral; Turkozelotes kazachstanicus: male palp: 9 - ventral, 10 - retrolateral; Zelotes prishutovae male palp: 11 -ventral, 12 - retrolateral. Scale bars: fig. 8 $1 \mathrm{~mm}$; figs. 6-7, 9-14-0.1 mm. 
Family Linyphiidae

Sintula spiniger (Balogh, 1935)

Material examined. Donetsk Reg., $1 \mathrm{~km}$ NW Kryva Luka, top of a chalk hill with sparse vegetation, 08.09.-10.10.2019, 1 ᄋ.

Distribution. From the Czech Republic and Austria to the South of European Russia (Rostov-on-Don Reg.) (Ponomarev, 2017; Nentwig et al., 2021). Ukraine: Kyiv Reg.: Kyiv (Singaevsky \& Balan, 2010), Kyiv (Pushcha Vodytsia), Kopachiv (Gnelitsa, 2012); Lugansk Reg.: Stanytsia-Luhanska Distr., Luhansk Natural Reserve (Gnelitsa, 2012; Polchaninova \& Prokopenko, 2013); Donetsk Reg. (first record).
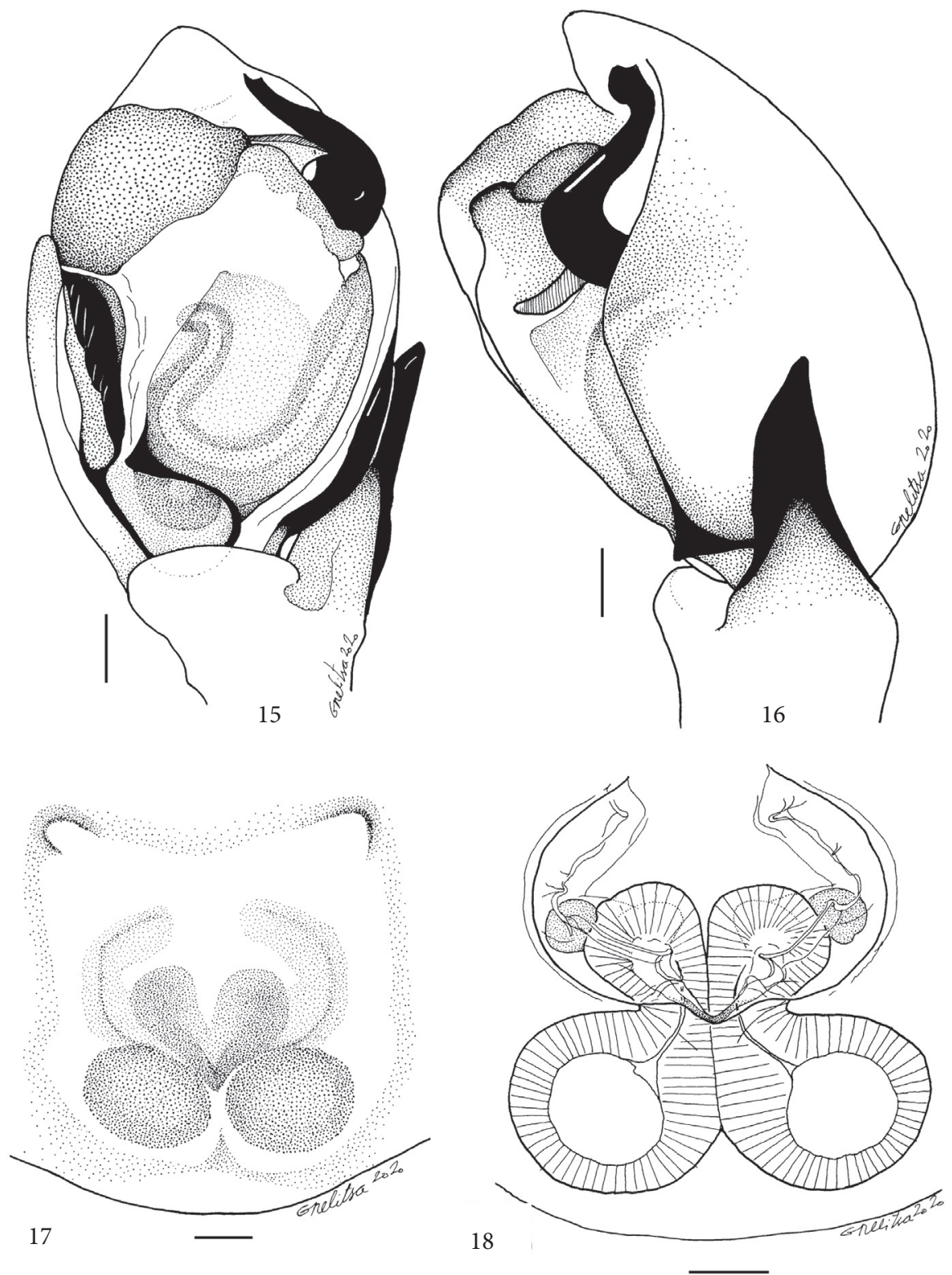

Figs 15-18. Zelotes pseudogallicus: male palp: 15 - ventral, 16 - retrolateral; 17 - epigyne ventral, 18 - vulva. Scale bars $0.1 \mathrm{~mm}$. 


\section{Family Lycosidae \\ Alopecosa inquilina (Clerck, 1757)}

Material examined. Kharkiv Reg., 4 km E Krasnokutsk, young pine plantation, 23.05-16.06.2019, 1 \& (R. Honcharov); idem, 04.07.-10.08.2019, 2 @ (R. Honcharov).

Distribution. Transeurasian species (WOS, 2021). Ukraine: Chernivtsi Reg. (as Bukovina in Roșca, 1937); Zakarpatska Reg. (Polozhentsev \& Akimtseva, 1980); Lviv, Ivano-Frankivsk Reg. (Legotay \& Tarasyuk, 1964); Kharkiv Reg. (first valid record).

Note. The following records were excluded as doubtful from the regional lists due to lack of collection material. Kharkiv Reg.: Chervona Khvylia; Kherson Reg.: Lower Dnipro (Polchaninova \& Prokopenko, 2013); AR Crimea: Simferopol (Kovblyuk \& Kastrygina, 2015). Thus, this is the first valid record from Left-Bank Ukraine.
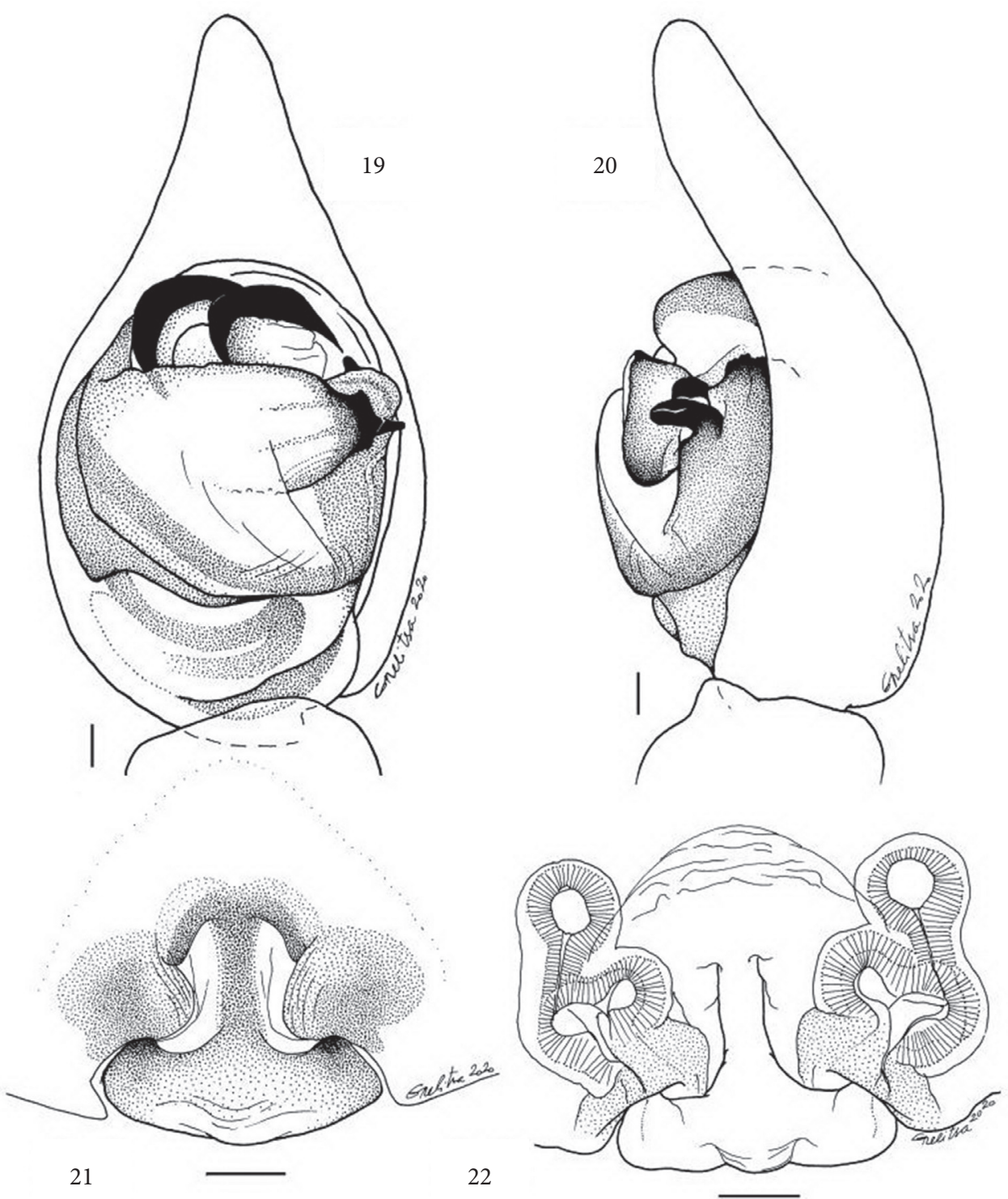

Figs 19-22. Alopecosa steppica: male palp: 19 - ventral, 20 - retrolateral; 21 - epigyne ventral, 22 - vulva. Scale bars $0.1 \mathrm{~mm}$. 
Alopecosa steppica Ponomarev, 2007 (fig 19-23)

Material examined. Donetsk Reg., $800 \mathrm{~m}$ SW Kryva Luka, pine forest on a chalk hill, 02.06-05.07.2019, 2 o'; idem, 02.06-05.07.2019, 13 ○; 1.3 km NE Kryva Luka, steep slope of a chalky riverbank, 02.06-05.07.2019,

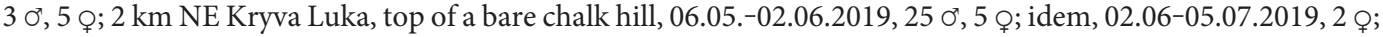
idem, edge of the Caragana thickets on a chalk hill, 06.05.-02.06.2019, 2 ơ, 1 o, 02.06-05.07.2019, 1 . .

Distribution. From Eastern Ukraine (Polchaninova \& Prokopenko, 2019) to Northern Kazakhstan (Kostanay Reg.) (Ponomarev et al., 2017 b) and southward to the Caspian Sea coast within Dagestan (Russia) (Ponomarev et al., 2011). Ukraine: Donetsk Reg. (Polchaninova \& Prokopenko, 2019): Dronivka, Saur-Mohyla (E. Prokopenko pers. comm.).

Note. The three known localities in Ukraine outline the western limit of the species range.

\section{Arctosa figurata (Simon, 1876)}

Material examined. Kharkiv Reg., 4 km E Krasnokutsk, bank of a bog, 23.05.-16.06.2019, 2 o".

Dist ribution. European species (Nentwig et al., 2021; WOS, 2021). Ukraine: Chernivtsi Reg.: Chernivtsi, Koroviya (Roşca, 1930), as Bucovina in Roşca, 1936; Lviv Reg.: Chervone, Stinka, Holohirky (Hirna, 2014), Lviv, Ivano-Frankivsk Reg. (Legotay \& Tarasyuk, 1964); Zhytomyr Reg.: Polissia Nature Reserve (Guryanova, 1989); Kharkiv Reg. (first record).

Note. First record from Left-Bank Ukraine.

\section{Piratula uliginosa (Thorell, 1856)}

Material examined. Kharkiv Reg., 4 km E Krasnokutsk, bank of a bog, 23.05.-16.06.2019, 1 o'.

Distribution. Europe (Nentwig et al., 2021), West Siberia (Mikhailov, 2013). Ukraine: Chernivtsi Reg.: as Bukovina in Koch, 1870; Lviv Reg.: Roztochchia, Kniazhe, Chervone (Petrusewicz, 1938), Rava-Ruska (Nowicki, 1870); Zhytomyr Reg.: Polissia Nature Reserve (Guryanova, 1989); Volyn Reg.: Shatskyi NNP (Evtushenko, 2013); Rivne Reg.: Bushcha (Hirna, 2015); Kyiv and Chernihiv reg: Chernihivske Polissia (Pochaninova \& Prokopenko, 2013); Kharkiv Reg. (first record).

\section{Family Mimetidae}

\section{Ero koreana Paik, 1967}

Material examined. Donetsk Reg., 2 km NE Kryva Luka, edge of the Caragana thickets on a chalk hill, 02.06.-05.07.2019, 1 ơ; Zaporizzhia Reg., Syvashyk, saline marshes, 10-22.06.2019, 1 o'.

Distribution. From Ukraine (Polchaninova \& Prokopenko, 2019) to Japan (WOS, 2021). Ukraine: Dnipropetrovsk Reg.: Kryvyi Rih (Evtushenko \& Isupova, 2013), Dnipro (Pochaninova \& Prokopenko, 2013); Donetsk Reg.: Siedove (Pochaninova \& Prokopenko, 2013, 2017); Mykolaiv Reg.: Yelanets Distr. (Polchaninova, 2021); (Zaporizhzhia Reg. (first record).

Note. Kryva Luka Vill. (Donetsk Reg.) is located on the northern boundary of the species range; Yelanetskyi Steppe Natural Reserve in Mykolaiv Reg. (Polchaninova, 2021) is the westernmost known locality.

\section{Family Philodromidae}

\section{Thanatus mongolicus (Schenkel, 1936)}

Material examined. Zaporizhzhia Reg., 2 km E Davydivka, saline marshes, 10-24.06.2019, 1 ơ.

Distribution. From Southern Ukraine (Polchaninova \& Prokopenko, 2013; Kovblyuk \& Kastrygina, 2015) to Hebei Province of China (Song et al., 2001). Ukraine: AR Crimea: Saky Distr. (Kovblyuk \& Kastrygina, 2015); Kherson Reg.: Rybalche (Polchaninova \& Prokopenko, 2013); Donetsk Reg. (Polchaninova \& Prokopenko, 2019): Siedove (E. Prokopenko, pers. comm.); Zaporizhzhia Reg. (first record). 
Note. Rybalche Vill. (Kherson Reg.) is the westernmost known locality. The northern boundary of the European part of the species range runs along the the northen coasts of the Balck Sea and the Sea of Azov to the Ergeni Upland.

\section{Family Salticidae}

Pellenes allegrii Caporiacco, 1935

Material examined. Kherson Reg., $1.2 \mathrm{~km}$ NNE Respublikanets, high riverbank dominated by forbs, 23.05.-22.06.2019, 1 ơ, 1 ᄋ (Polchannova \& Iosypchuk); 500 m SSE Respublikanets, medium-height riverbank dominated by forbs, 23.05.-22.06.2019, 1 O', 1 \% (Polchannova \& Iosypchuk).

Distribution. From Southern Ukraine (Polchaninova \& Prokopenko, 2019; Iosypchuk, 2019) to Uzbekistan, Kyrgyzstan (Mikhailov, 2013), Northern India and Pakistan (Caporiacco, 1935). Ukraine: Donetsk Reg. (Polchaninova \& Prokopenko, 2019): Siedove, Bilosaraiska spit (E. Prokopenko, pers. data); Kherson Reg.: Respublikanets (Iosypchuk, 2019).

Note. Respublikanets Vill. (Kherson Reg.) is the westernmost known locality.

\section{Family Theridiidae}

\section{Neottiura suaveolens (Simon, 1879)}

Material examined. Donetsk Reg., $1.3 \mathrm{~km}$ NE Kryva Luka, edge of the Caragana thickets on a chalk hill, 31.05.2019, 1 \&.

Distribution. Europe (Nentwig et al., 2021), Caucasus (North Georgia) (Ponomarev \& Komarov, 2015), South Urals (Esyunin \& Efimik, 1996), South CisUrals (Orenburg Reg.) (Esyunin et al., 2007). Ukraine: Khmelnytsk Reg.: Panivtsi (Guryanova, 2003); Mykolaiv Reg.: Kurypchine (Polchaninova et al., 2017); Kharkiv Reg. (Polchaninova \& Prokopenko, 2019): Zelenyi Hai (N. Polchaninova pers. data); Luhansk Reg.: Krynychne, Horodyshche (Polchaninova \& Prokopenko, 2013); Donetsk Reg. (first record).

Note. Only five localities are known in the East European Plain (all in Ukraine); the South Urals and CisUrals are the areas detached from the main species range (Esyunin \& Efimik, 1996; Esyunin et al., 2007).

\section{Family Thomisidae \\ Bassanioides caperatus (Simon, 1875) (figs 24-28, 32-33)}

Material examined. Zaporizhzhia Reg., $4.8 \mathrm{~km} \mathrm{NE}$ Sheliuhy, sandy steppe with clamshells, 07-24.06.2019, 1 o; 1.5 km NEE Nove, grazed abandoned field, 10-22.06.2019, 1 o; idem, saline marshes, 10-22.06.2019, 1 ᄋ.

Distribution. From Spain (Nentwig et al., 2021) to Southern European Russia (Krasnodar Krai) (Spassky, 1937). Ukraine: AR Crimea: Saky, Sevastopol, Simferopol, Sudak, Feodosiya, Yalya distr; Tarkhankut (Kovblyuk \& Kastrygina, 2015; Nadolny, 2020); Zaporizhzhia Reg. (first record).

Note. The record "Khorly, Kherson Area" requires verification (see Polchaninova \& Prokopenko, 2017); therefore, the material from Zaporizhzhia Region is the first valid record from Left-Bank Ukraine. The northern boundary of the species range runs along the northern coast of the Sea of Azov.

\section{Heriaeus horridus Tyschchenko, 1965 (figs 27-28, 34)}

Material examined. Kherson Reg., 1,85 km SW Shyroka Balka, loess slope, 10.06-3.07.2020, 3 ơ (Iosypchuk); Zaporizhzhia Reg., 1.7 km E Davydivka, sandy steppe, 07-24.06.2019, 1 ơ; 2 km E Davydivka, saline marshes, 07-24.06.2019, 1 ơ; 1.5 km Nove, saline marshes, 10-22.06.2019, 15 ơ; $1.3 \mathrm{~km}$ E Nove, grazed abandoned field, 10-22.06.2019, 1 \% .

Distribution. From Southern Ukraine to West Siberia and East Kazakhstan (Mikhailov, 2013), southward to Northern India (Uttharakhand State) (Tikader, 1980). Ukraine: AR Crimea: Tarkhankut peninsula (Nadolny, 2020); Kherson and Zaporizhzhia reg. (first records). 
Note. First record from mainland Ukraine. Shyroka Balka vill. in Kherson Region is the westernmost known locality.

Ozyptila lugubris (Thorell, 1875) (figs. 29-30)

Material examined. Donetsk Reg., 800 m SW Kryva Luka, bare chalk slope, 08.09.2019, 1 ơ.

Distribution. From Southern Ukraine (Deli, 2012) to Eastern Kazakhstan; south to the Caucasus and Turkey (Marusik \& Mikhailov, 2021). Ukraine: Odesa Reg.: Lover Danube region (Deli, 2012 as O. lugubris); AR Crimea: Saki, Sevastopol, Simferopol, Sudak, Feodosiya, Yalta distr. (Kovblyuk \& Kastrygina, 2015 as O. lugubris); Donetsk Reg. (first record).

Note. First record from Left-Bank Ukraine.

Xysticus mongolicus Schenkel, 1963 (fig. 24)

Material examined. Donetsk Reg., $1.3 \mathrm{~km}$ NE Kryva Luka, edge of the Caragana thickets on a chalk hill, 08.09.-10.10.2019, 1 o'.

Distribution. From Serbia (Nentwig et al., 2021) to China (Inner Mongolia) (Song, et al., 1999). Ukraine: Kharkiv Reg.: Kytsivka, Krasne Pershe; Dnipropetrovsk Reg.: Obukhivka (as Kirovske); Kherson Reg.: Novochornomorya (Polchaninova, 2013, 2019; Polchaninova \& Prokopenko, 2013, 2017); Luhansk Reg. (Polchaninova \& Prokopenko, 2019): Stanytsia-Luhanska Distr., 'Pryazovska Poima' department of the Luhansk Natural Reserve (E. Prokopenko pers. data); Donetsk Reg. (first record).

Note. Kharkiv Region lies on the northern boundary of the species range.

\section{Family Titanoecidae}

\section{Titanoeca ukrainica Guryanova, 1992}

Material examined. Kherson Reg., $1.2 \mathrm{~km}$ NNE Respublikanets, high clayey riverbank dominated by Galatella villosa (L.) Rchb. f. 1853, 23.05-22.06.2019, 2 ơ (Polchaninova \& Iosypchuk).

Distribution. From Southern Ukraine (Kovblyuk \& Kastrygina, 2015) to Southern European Russia (Rostov-on-Don Reg.) (Ponomarev, 2017). Ukraine: Kherson Reg.: Askania-Nova (Polchaninova \& Prokopenko, 2013, 2019), Respublikanets (Iosypchuk, 2019); AR Crimea: Simferopol Distr.; Tarkhankut peninsula (Kovblyuk \& Kastrygina, 2015; Nadolny, 2020).

Note. Respublikanets Vill. (Kherson Reg.) lies on the northern boundary of the species range.

\section{Family Zodariidae}

Zodarion morozum Denis, 1935

Material examined. Zaporizhzhia Reg.: 5 km W Stepanivka, mesic saline meadow, 08-23.06.2019, 2 O’.

Distribution. Albania, North Macedonia, Greece, Bulgaria, Turkey (Nentwig et al., 2021), South of European Russia (from the coast of the Sea of Azov to the western coast of the Caspian Sea) (Ponomarev et al., 2011; Ponomarev \& Dvadnenko, 2012). Ukraine: Odesa Reg.: Lover Danube region (Deli, 2012); AR Crimea: Bakhchysarai, Lenino, Saky, Sevastopol, Simferopol, Feodosiya, Yalta distr.; Tarkhankut peninsula (Kovblyuk \& Kastrygina, 2015; Nadolny, 2020); Zaporizhzhia Reg. (first record).

Note. First record form Left-Bank Ukraine. The northern boundary of the species range runs through the coastal areas of the Black Sea and the Sea of Azov. 


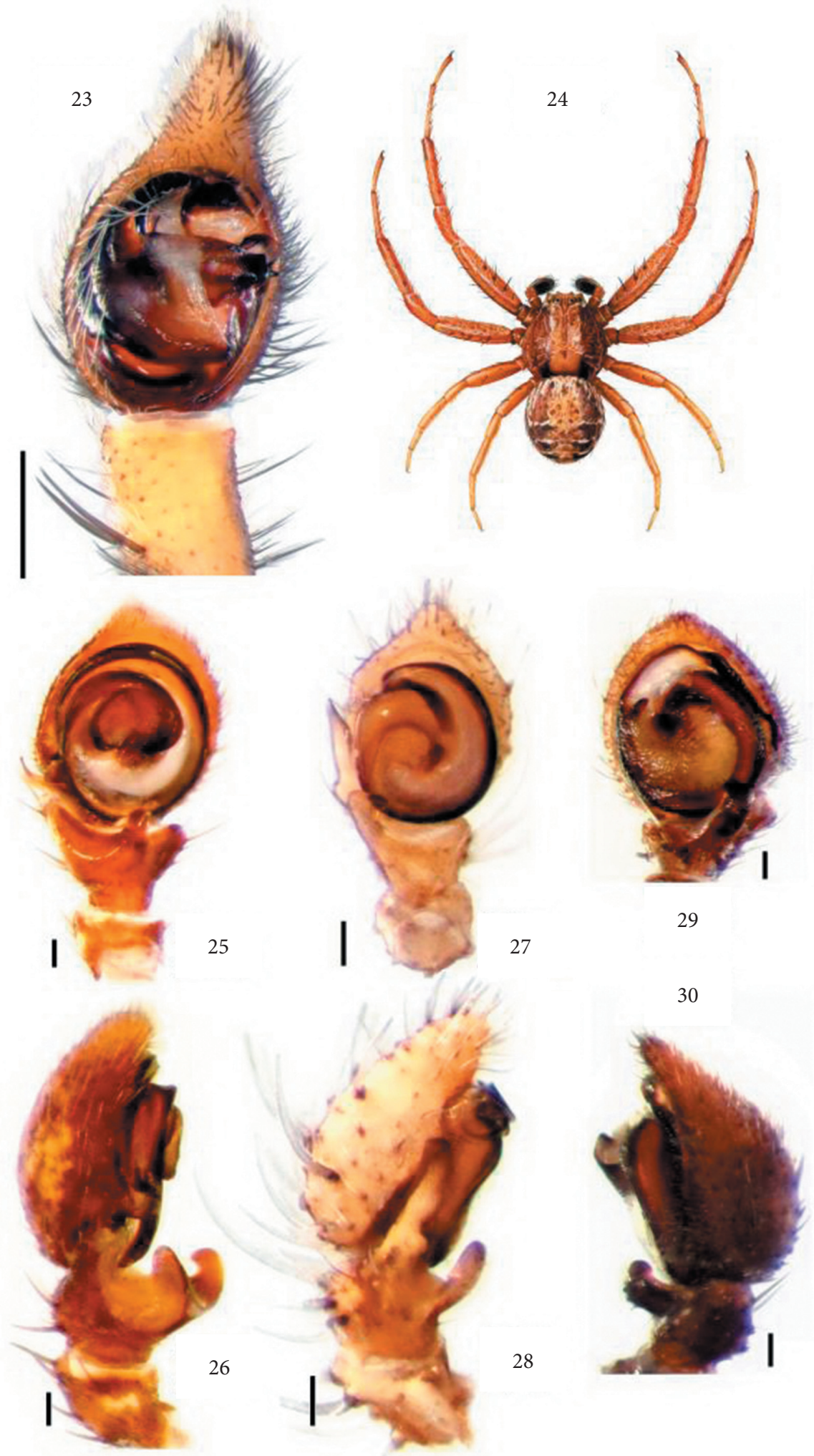

Figs 23-30. Alopecosa steppica: male palp: 23 - ventral; Xysticus mongolicus: 24 - general appearance dorsal (after Polchaninova 2013, drawing by V. Timokhanov); Bassanioides caperatus: male palp: 25 - ventral, 26 retrolateral; Heriaeus horridus: male palp: 27 — ventral, 28 - retrolateral; Ozyptila tuberosa: male palp: 29 ventral, 30 - retrolateral. Scale bars $0.1 \mathrm{~mm}$. 


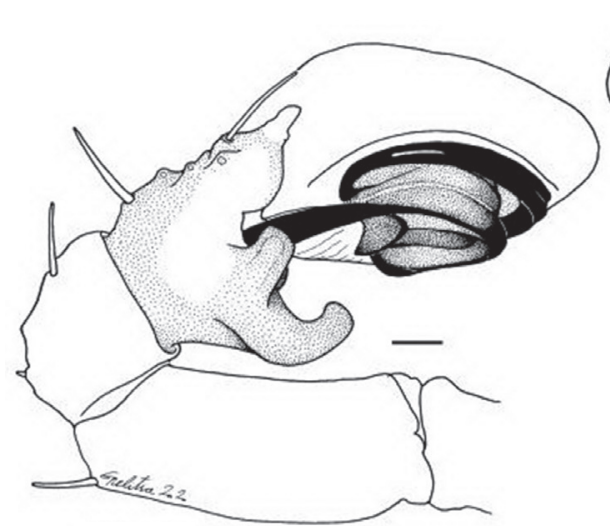

31

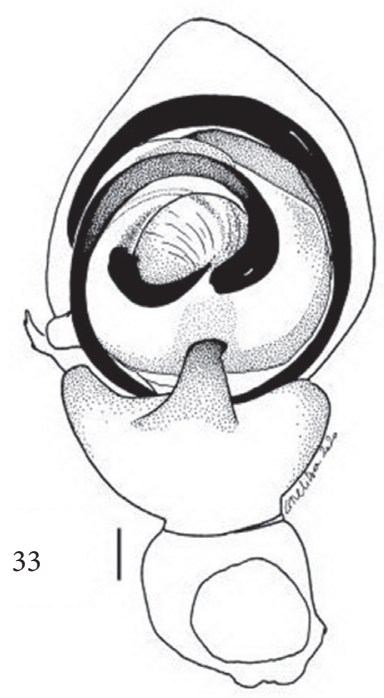

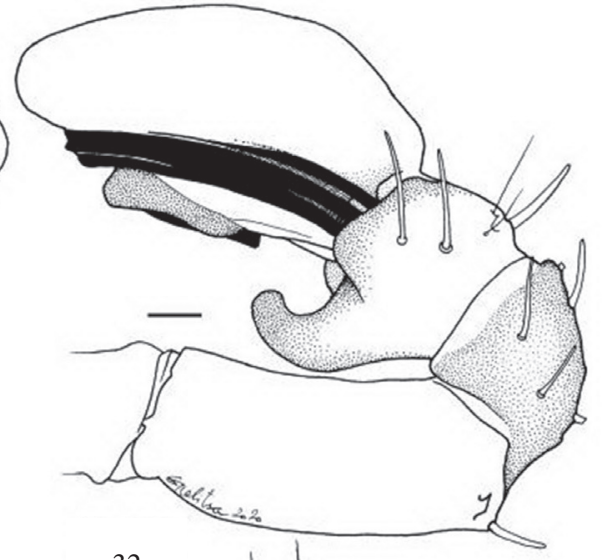

32

34

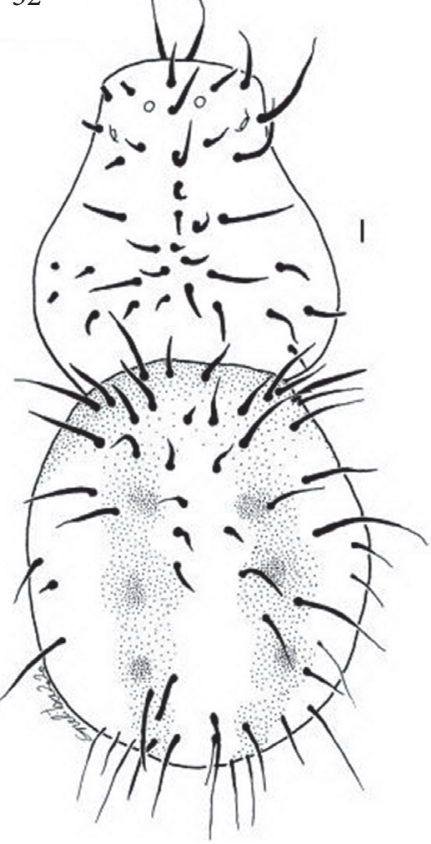

Figs 31-34. Bassanioides caperatus: male palp: 31 - retrolateral, 32 - prolateral, 33 - ventral; Heriaeus horridus: 34 - general appearance dorsal. Scale bars $0.1 \mathrm{~mm}$.

\section{Discussion}

Out of 31 species listed in this paper, the four are new to Ukraine: Zelotes atrocaeruleus, Marinarozelotes manychensis and Turkozelotes kazachstanicus were collected in the south of Zaporizhzhia Region, while Zelotes puritanus in the north of Kharkiv Region. Nine species (Clubiona genevensis, Dictyna sinuata, Micaria coarctata, Zelotes erebeus, Zelotes prishutovae, Arctosa figurata, Heriaeus horridus, Ozyptila tuberosa, and Zodarion morozum) were recorded for the first time from Left-Bank Ukraine. For Heriaeus horridus, this is the first record form mainland Ukraine. Three species (Drassyllus crimeaensis, Bassanioides caperatus and Alopecosa inquilina) were previously excluded from the list of spiders of Left-Bank Ukraine due to the lost of collection material (Polchaninova \& Prokopenko, 2019). Now they are restored, since new data proved their occurrence. Accounting new records, 1081 spider species are currently known in the Ukrainian fauna, and 757 species in the fauna of Left-Bank Ukraine. The regional lists were replenished with four species 
in Donetsk Region, seven species in Kharkiv Region, one species in Kherson Region, and 15 species in Zaporizhzhia Region.

Our research outlines newboundaries of Marinarozelotes manychensis and Turkozelotes kazachstanicus, distribution and expand them to the west of Rostov-on-Don Region (Russia) to Zaporizhzhia Region (Ukraine). Micaria coarctata is recorded from the East European Plain for the first time; the occurrence of Drassyllus crimeaensis and Bassanioides caperatus was confirmed for the coastal areas of the south of the East European Plain.

We have clarified distribution limits of some species inhabiting European steppes and saline marshes. The northern boundary of Zodarion morozum range runs along the the Black Sea and the Sea of Azov nothern coasts. Some Central-Asian species (Devade tenella, Thanatus mongolicus, Xysticus mongolicus) have western limits of their distribution in the left-bank part of the Lower Dnipro region. Some species are spread from the Crimea and the northwestern coast of the Sea of Azov to West Kazakhstan (Talanites strandi) or to South Siberia and Turkmenistan (Gnaphosa ukrainica). The known range of Heriaeus horridus stretches from the right-bank of the Dnipro estuary to Eastern Kazakhastan and Northern India. Alopecosa steppica and Zelotes pseudogallicus ranges are delimited by Eastern Ukraine in the west and by West Siberia (Kostanay Reg. of Kazakhstan and/or Tyumen Reg. of Russia) in the east. Interestingly, the Pryazovskyi National Park gave a number of unexpected records, although the spider faunas of the Azov Sea coast on the Bilosaraiska spit (Donetsk Region) and the Black Sea coast on the Kinburn peninsula (Kherson Region) situated east and west of Zaporizhzhia Region had been studied well (Polchaninova, 2012; Ponomarev et al., 2016).

Investigation of the bogs and pine forests in the Slobozhanskyi National Park in the north of Kharkiv Region gave records of another group of species with Transpalearctic (Alopecosa inquilina), disjunctive (Callilepis shutzeri), European-West Siberian (Pardosa uliginosa), and European (Arctosa figurata) ranges. Their typical habitats are forests, floodplain meadows, wetlands and, in case of A. figurata (Polchaninova, 2020), meadow steppes. They were found in the Wood-and-Steppe zone of Left-Bank Ukraine for the first time.

We are thankful to R. Honcharov (Kharkiv), K. Orlova-Hudim (Kherson), and H. Mykytynets (Melitopol) for the help in collecting the material. Special thanks go to H. Mykytynets for organizing the field trips. We are also grateful to A. Motliakh (Slobozhsnsky NNP), S. Lymanskyi ('Kreidova Flora' Department, Ukrainian Steppe Natural Reserve), and A. Lopushynskyi (NNP 'Kamyanska Sich'), for the access to the protected areas and favorable working conditions. We really appreciate the help of A. Ponomarev (Rostov-on-Don) and E. Prokopenko (Donetsk), who provided the data from their personal collections, and the valuable advice on photography techniques by G. Azarkina (Novosibirsk) and M. Kovblyuk (Simferopol). The critical comments on the initial draft by S. Esyunin (Perm) and Yu. Marusik (Magadan), as well as the suggestions of the anonymous reviewers are also deeply acknowledged.

\section{References}

Azarkina, G. N., Lyubechanskii, I. I., Trilikauskas, I. A., Dudko, R. Y., Bespalov, A. N., Mordkovich, V. G. 2018. A checklist and zoogeographic analysis of the spider fauna (Arachnida: Aranei) of Novosibirsk Area (West Siberia, Russia). Arthropoda Selecta, 27 (1), 73-93. doi:10.15298/arthsel.27.1.11

Caporiacco, L. di. 1935. Aracnidi dell'Himalaia e del Karakoram, raccolti dalla Missione italiana al Karakoram (1929-VII). Memorie della Società Entomologica Italiana, 13, 161-263

Deli, O. F. 2012. Checklist of spiders (Araneae) of Lower Danube region of Ukraine. Visnyk Odeskoho Natsionalnoho Univiversitetu. Seria Biologia, 17, 96-104 [In Ukrainian].

Demir, H., Seyyar, O., Türker, H., Koçyiðt, H.O. \& Öner, H. 2015. The spider fauna of Melendiz Mountains, Nigde, Turkey. Serket, 14 (3), 146-166.

Esyunin, S. L., Efimik, V. E. 1996. Catalogue of spiders (Arachnida, Aranei) of the Urals. Ed. K. G. Mikhailov. KMK Scientific Press, Moscow, 1-220.

Esyunin, S. L., Sozontov, A. N. 2016. On a new Eurasian species of Dictyna Sundevall, 1833 (Aranei: Dictynidae), with taxonomic notes on poorly known Palearctic Dictyna species. Arthropoda Selecta, 25, 199-206.

Esyunin, S. L., Tuneva, T. K. 2020. A review of the family Gnaphosidae in the fauna of the Urals (Aranei), 6. Taxonomic remarks and new records, with description of a new species. Arthropoda Selecta, 29, 103-120. https://doi.org/10.15298/arthsel.29.1.09 
Esyunin, S. L., Tuneva, T. K., Farzalieva, G. S. 2007. Remarks on the Ural spider fauna (Arachnida: Aranei). 12. Spiders of the steppe zone of Orenburg Region. Arthropoda Selecta, 16, 43-63.

Evtushenko, K. V. 1991. Ecological and faunistic studies of spiders (Aranei) in Kievskoye Polessie. Editorial board of 'Vestnik Zoologii'. Deposit in VINITI 04.06.91. N 2338-B91.

Evtushenko, K. V. 2013. Structure of spider assemblages (Aranei) of the above-soil layer of four typical habitats of the 'Shatskyi' National Nature Park. Pryroda zakhidnoho Polissia ta prylehlykh terytorij, 10, 124-130 [In Ukrainian with English summary].

Evtushenko, K. V., Isupova, N. N. 2013. Spiders (Aranei) of the birch forests of the Kryvyi Rih, In: Akimov, I. A., ed., Tezy dopovidei VIII Zjizdy HO "Ukrainske entomolohishne tovarystvo", 26-30 September 2013, Kyiv, 49-50.

Gnelitsa, V. A. 2012. The genus Sintula (Aranei, Linyphiidae) in Ukraine, with the description of a new species. Vestnik Zoologii, 46, 16-23.

Guryanova, V. Y. 1989. Spiders (Aranei) of the near-soil stratum of the Polessye State Reserve, Vestnik Zoologii, 2, 6-12 [In Ukrainian with English summary].

Guryanova, V. Y. 2003. Materials to the sider fauna of Podolian Wood-and-Steppe (Ukraine). Vestnik Zoologii, 35, 3-11 [In Ukrainian with English summary].

Hirna, A. 2014. A checklist of spiders of the Hologory district within Roztochia-Opillia Hillsregion (Northwestern Podolia). Naukovi osnovy zberezhennia biotopichnoho riznomanottia, 5, 91-144 [In Ukrainian with English summary].

Hirna, A. 2015. Specimens of spider fauna from Ukraine in the collection of the Museum of Natural History, Wroclaw University (According to the collection of Stanisław Pilawski and Kazimierz Petrusewicz). Zoologica Polonica, 60, 15-33.

Iosypchuk, A. M. 2019: First data on the spider fauna (Araneae) of the National Nature Park 'Kamianska Sich' (South Ukraine). In: Akulov, A. V. et al., eds. From a Molecula to the Biosphere. Abstract of XIV International Young Scientists' Conference (November 27 ${ }^{\text {th }}-29$ th, 2019), Kharkiv, 169-171.

Iosypchuk, A. M., Polchaninova, N. Yu., Orlova-Hudim K. S. 2020. New data on the spider (Araneae) species composition and habitat distribution of the National Nature Park 'Dzharylhatskyi'. Scientific Bulletin of Natural Sciences (Biological Sciences). Kherson, 28, 44-52. doi: 10.32999/ksu2524-0838/2020-28-4 [In Ukrainian].

Koch, L. 1870. Beiträge zur Kenntniss der Arachnidenfauna Galiziens. Jahrbuch der Kaiser.-Königshen Gelehrt und Gesellschaft in Krakau. 41, 1-56.

Kovblyuk, M. M., Kastrygina, Z. A. 2015. Updated catalogue of the spiders (Arachnida, Aranei) of the Crimea. Ukrainska Entomofaunistyka, 6, 1-81.

Kovblyuk, M. M., Seyyar, O., Demir, H., Topcu, A. 2009. New taxonomic and faunistic data on the gnaphosid spiders of Turkey (Aranei: Gnaphosidae). Arthropoda Selecta, 18, 169-187.

Krasnobayev, Yu. P. 2007. Oder Aranei (Spiders). In: Rosenberg, G. S., ed. Cadaster of invertebrates of Samarskaya Luka. Tutorial. OOO “Ofort”, Samara, 79-93 [In Russian].

Legotay, M. V., Tarasyuk, G. D. 1964. Ecological distribution of the araneofauna of Ciscarpathia, In: Ekologia nasekomykh $i$ drygikh bespozvonochnykh Sovietskikh Karpat. Mater. mezhvuzovskoi konferentsii. Uzhgorodskiy universitet, Zakarpatskiy fililial VEO, Uzhgorod, 54-59 [In Russian].

Li, S., Lin, Y. 2016. Species catalog of China, Vol. 2. Animals. Invertebrates (I). Arachnida: Araneae. Science Press, Beijing, 1-549.

Marusik, Yu. M., Mikhailov, K. G. 2021. Revalidation of Xysticus tuberosus Thorell,1875 (Aranei: Thomisidae) with notes on the related species. Arthropoda Selecta, 30, 119-124. doi: 10.15298/arthsel.30.1.11.

Marusik, Yu. M., Omelko, M. M. 2018. A survey of the Porrhoclubiona Lohmander, 1944 from Central Asia (Araneae, Clubiondae). ZooKeys, 802, 19-38. doi:10.3897/zookeys.802.30236

Matveev, V. A., Krasnobaev, Yu. P., Bekmansurova, E. V. 2003. Fauna and ecology of spiders of the Mari-El Republic. Marijskij gos. un-t, Zhigulevskij gos. prirodnyj zapovednik im. I. I. Sprygina, Samara, 1-86 [In Russian].

Mikhailov, K. G. 2013. The spiders (Arachnida: Aranei) of Russia and adjacent countries: a non-annotated checklist. Arthropoda Selecta, Supplement 3, 1-260.

Mikhailov K. G. 2021. Advances in the study of the spider fauna (Aranei) of Russia and adjacent regions: a 2017 update. Invertebrate Zoology, 18, 25-35.

Nadolny, A. A. 2020. New data on the species composition of spiders (Arachnida: Aranei) in Tarkhankut Peninsula, Crimea. Proceedings of T. I. Vyazemsky Karadag scientific station - Natural reserve of the RAS, 3(15), 29-60 [In Russian].

Nentwig, W., Blick, T., Gloor, D., Hänggi, A., Kropf, C. 2021. Araneae. Version 02.2021. https://www.araneae. nmbe.ch (accessed 13.02.2021)

Nowicki, M. 1870. Zapiskifaunicze. Sprawozdanie Komisji Fizjograficznej, Kraków, 4, 1-30.

Petrusewicz, K. 1938. Badania pająkow na polnocnei Kraweddzi Podola. I. Pisautida, Lycosidae, Argiopidae. KOSMOS. Seria A. Rozpravy, 63, 317-362.

Piterkina, T. V., Ovtcharenko, V. I. 2009. Fauna and ecology of gnaphosid spiders (Aranei: Gnaphosidae) in clay semidesert of Western Kazakhstan. Arthropoda Selecta, 17, 175-184. 
Polchaninova, N. Yu. 2012. A checklist of spiders (Araneae) of the Chornomorskyi Biosphere Reserve (Ukraine). Pryrodnychyi Almanakh. Biolohichni nauky, 18. PAT "Khersonska miska drukarnia”, Kherson, 85-108.

Polchaninova, N. Yu. 2013. Xysticus mongolicus Schenkel, 1863. In: Tokarsky, V. A., chief ed., Chervona Knyha Kharkivskoi Oblasti. Tvarynnyi Svit. KhNU im. V. N. Karazina, Kharkiv, 53.

Polchaninova, N. 2019. Rare spider species (Araneae) of protected steppe areas of the Kharkiv Region (Ukraine). The Journal of V. N. Karazin Kharkiv National University. Series "Biology", 32, 99-106. DOI: 10.26565/2075-5457-2019-32-12

Polchaninva, N. 2020. Spiders (Aranei) of the 'Privolzhskaya Lesostep' Nature Reserve (Penza Area, Russia) the sector 'Kuncherovskaya Lesostep'. Arthropoda Selecta, 29 (3), 371-386. DOI: 10.15298/arthsel. 29.3.11

Polchaninova, N. 2021. Spiders (Arachnida: Araneae) in dry grasslands of South Ukraine: a case study of Yelanetskyi Steppe Natural Reserve. Arachnologische Mitteilungen, 61, 27-35. DOI: 10.30963/ aramit6105

Polchaninva, N. Yu., Gnelitsa, V. A., Evtushenko, K. V., Singaevsky, E. N. 2017. An annotated checklist of spiders (Arachnida: Aranei) of the National Nature Park 'Buzkyi Hard' (Mykolaiv Area, Ukraine). Arthropoda Selecta, 26 (1), 253-272.

Polchaninva, N., Krasova, O., Lysogor, L. \& Atemasova T. 2021. Assessment of the conservation value of dry grassland habitats in the Inhulets River basin (Central Ukraine) based on vegetation and spider research. Hacquetia, 20 (1), 225-242. DOI: 10.2478/hacq-21-003

Polchaninova, N. Yu., Prokopenko, E. V. 2013. Catalogue of the spiders (Arachnida, Aranei) of Left-Bank Ukraine. Arthropoda Selecta, Supplement 2, 1-268.

Polchaninova, N. Yu., Prokopenko, E. V. 2017. Catalogue of the spiders (Arachnida, Aranei) of Left-Bank Ukraine. Addendum 1. 2013-2016. Mikhailov, K. G., ed. Arthropoda Selecta, Supplement 4, 1-115.

Polchaninova, N., Prokopenko, E. 2019. An updated checklist of spiders (Arachnida: Araneae) of Left-Bank Ukraine. Arachnologische Mitteilungen, 57 (1), 60-64. doi: 10.30963/aramit5711

Polozhentsev, P. A., Akimseva, N. A. 1980. Spiders of forest biocenosis of Transcarpathia. Entomologicheskoye obozreniye, 59, 448-450 [In Russian with English summary].

Ponomarev, A. V. 2008. The additional data to the spider fauna of the south-east of the Russian Plain. Vestnik Yuznogo Nauchnogo Tsentra, Ros. Akad. Nauk, Rostov, 4, 78-86 [In Russian with English summary].

Ponomarev, A. V. 2017. Spiders (Arachnida: Aranei) of steppe and meadow-steppe habitats of gully and ravine ecosystems of the valley of the Don River lower reaches. Proceedings of the Russian Entomological Society, 88, 118-131 [In Russian with English summary].

Ponomarev, A. V., Abdurakhmanov, G. M., Alieva, S. V., Dvadnenko, K. V. 2011. Spiders (Arachnida: Aranei) of the coasts and islands in Northern Dagestan. South of Russia: ecology, development, 4, 126-143 [In Russian with English summary].

Ponomarev, A. V., Alekseev, S. A., Kozminykh, V. O., Shmatko, V. Yu. 2017 a. Spiders (Arachnida; Aranei) of Stavropol Province of Russia. Arthropoda Selecta, 26, 155-173.

Ponomarev, A. V., Aliev, M. A., Khabiev, G. N., Shmatko, V. Yu. 2019. New data on the spider fauna (Aranei) of Dagestan, Russia. Arthropoda Selecta, 28, 309-334. doi:org/10.15298/arthsel.28.2.14

Ponomarev, A. V., Bragina, T. M., Shmatko, V. Yu. 2017 b. New data on spiders (Aranei) of the Naurzum State Natural Reserve (Kostanay Region, Kazakhstan)]. Caucasian Entomological Bulletin, 13, 3-10 [In Russian with English summary]. doi:10.23885/1814-3326-2017-13-1-3-10

Ponomarev, A. V., Dvadnenko, K. V. 2011. A new species of the spiders genus Turkozelotes Kovblyuk et Seyyar, 2009 (Aranei: Gnaphosidae) from the Rostov region]. Vestnik Yuznogo Nauchnogo Tsentra, Ros. Akad. Nauk, Rostov, 7, 108-110 [In Russian with English summary].

Ponomarev, A. V., Dvadnenko K. V. 2012. Notes of the spider (Aranei) fauna and taxonomy of the south of Russia and West Kazakhstan. South of Russia: ecology, development, 7, 42-53 [In Russian with English summary].

Ponomarev, A. V., Khnykin A. S. 2013. Spiders (Aranei) of Volgograd City and its Environs. South of Russia: ecology, development, 8, 109-136 [In Russian with English summary].

Ponomarev, A. V., Komarov Yu. A. 2015. Spiders (Aranei) of the Republic of South Ossetia. South of Russia: ecology, development, 10, 116-147 [In Russian with English summary].

Ponomarev, A. V., Mikhailov, K. G., Shmatko V. Yu. 2021. Taxonomic remarks on the genus Turkozelotes Kovblyk et Seyyar, 2009 (Aranei: Gnaphosidae). Arthropoda Selecta, 30, 113-118. doi: 10.15298/ arthsel30.1.10

Ponomarev, A. V., Prokopenko E. V., Ivliev P. P., Shmatko V. Yu. 2016. Spiders (Aranei) of the coast of Taganrog Bay (the Sea of Azov) and the Don River delta. Caucasian Entomological Bulletin, 12, 3-28 [In Russian with English summary].

Ponomarev, A. V., Prokopenko E. V., Shmatko V. Yu. 2017 c. New and interesting records of spiders (Arachnida: Aranei) from the southeastern part of the Russian Plain. Proceedings of the Russian Entomological Society, 88, 103-117 [In Russian with English summary].

Ponomarev, A. V., Shapovalov, M. I., Ivliev, P. P. 2014. New data to the spider fauna (Aranei) of the south of the European part of Russia. Vestnik Adygeiskogo gosudarstvennogo universiteta, 2 (137), 54-60 [In Russian with English summary]. 
Ponomarev, A. V., Shmatko, V. Yu. 2019. A review of spiders of the genus Zelotes Gistel, 1848 of the subterraneusgroup (Aranei: Gnaphosidae) from the Caucasus and Ciscaucasia. Caucasian Entomological Bulletin, 15, 3-22 [In Russian with English summary] doi:10.23885/181433262019151-322

Ponomarev, A. V., Shmatko, V. Yu. 2020. A review of spiders of the genera Trachyzeloes Lohmander, 1944 and Marinarozelotes Ponomarev, gen. n. (Aranei: Gnaphosidae) from the southeast of the Russian Plain and the Caucasus. Caucasian Entomological Bulletin, 16, 125-139 [In Russian with English summary]. doi:10.23885/181433262020161-125139

Ponomarev, A. V., Mikhailov, K. G., Shmatko V. Yu. 2021. Taxonomic remarks on the genus Turkozelotes Kovblyk et Seyyar, 2009 (Aranei: Gnaphosidae). Arthropoda Selecta, 30, 113-118. doi: 10.15298/ arthsel30.1.10

Prokopenko, E. V., Chumak, V. A. \& Lachat, T. 2014. Ground-dwelling arachnids of a beech forest (Zakarpattia Region). Visnyk Dnipropetrovskoho Derzhavnoho Ahrarno-Ekonomicheskoho Univiversytetu, 33, 110-114 [In Russian with English summary].

Roşca, A. 1930. Contributiuni la Cunoasterea Arachnidelor din Bukovina. Buletin Facultatea de Ştiinţe, Cernăuţi, 4, 201-219.

Roşca, A. 1936. Fauna araneelor din Bucovina (sistematica, ecologia si raspandirea geographies). Buletin Facultatea de Ştiinţe, Cernăuţi, 10, 123-216.

Roşca, A. 1937. Suplement la fauna Araneelor din Bucovina. Buletin Facultatea de Ştiinţe, Cernăuţi, 11, $225-236$.

Rozwałka, R, Rutkowski, T, Sienkiewicz, P, Zawal, A. 2016. Zelotes erebeus (Thorell, 1871) (Araneae: Gnaphosidae) in Poland and its distribution in Europe. Entomologica Fennica, 27, 1-7.

Senglet, A. 2011. New species in the Zelotes tenuis-group and new or little known species in other Zelotes groups (Gnaphosidae, Araneae). Revue Suisse de Zoologie, 118, 513-559.

Singaevsky, E. M. 2014. Spiders (Arachnida, Aranei) in Middle Dnieper Ukraine: fauna and ecology. Thes. Cand. of Biol. Sci. (Ph.D.). I. I. Schmalhasen Zool. Inst. NAS of Ukraine, Kyiv, 1-14 [In Ukrainian with English summary].

Singaevsky, E. \& Balan, P. 2010. Arachnids (Arachnida: Acari (Parasitiformes: Mesostigmata); Aranei) of the green zones of Kyiv City and suburban. Visnyk Kyivskoho Natsionalnoho Univiversytetu im. Tarasa Shevchenko, 55, 42-45 [In Ukrainian with English summary].

Spassky, S. A. 1937. Materials to the spider fauna of the Black Sea coast. Sbornik nauchno-issledovatelskikh rabot Azovo-Chernomorskogo selkhozinstituta, 5, 131-138 [In Russian].

Spassky, S. A. 1940. Araneae palaearcticae novae. V. Folia Zoologica et Hydrobiologica, 10, 353-364.

Song, D. X., Zhu, M. S., Chen, J. 1999. The spiders of China. Hebei Science and Technology Publishing House, Shijiazhuang, 1-640.

Song, D. X., Zhu, M. S., Chen, J. 2001. The Fauna of Hebei, China: Araneae. Hebei Science and Technology Publishing House, Shijiazhuang, 1-510.

Tikader, B. K. 1980. Thomisidae (Crab-spiders). Fauna India (Araneae), 1, 1-47

Trilikauskas, L. A. 2013. On the fauna of spiders and harvestmen (Arachnida: Aranei et Opiliones) of Gornaya Shoria, South Siberia. Eurasian Entomological Journal, 12, 438-450 [In Russian with English summary].

World Spider Catalog (WOS). 2021. version 22. Bern: Natural History Museum. http://wsc.nmbe.ch (accessed 13.02.2021)

Zamani, A., Marusik, Yu. M. 2018. The first report on the spider fauna (Arachnida: Araneae) of the Lut Desert, Iran. Acta Arachnologica, 67, 67-75. doi:10.2476/asjaa.67.67

Zamani, A., Mirshamsi, O., Dolejš, P., Marusik, Y. M., Esyunin, S. L., Hula, V., Ponel, P. 2017. New data on the spider fauna of Iran (Arachnida: Araneae), part IV. Acta Arachnologica, 66 (2), 55-71. doi:10.2476/ asjaa.66.55

Zamani, A., Mirshamsi, O., Mohammadi Kashani, G., Karami, L. 2018. New data on the spider fauna of Iran (Arachnida: Araneae), part V. Iranian Journal of Animal Biosystematics, 13 (2, for 2017), 183-197. doi:10.22067/ijab.v13i2.72404

Received 5 December 2020

Accepted 3 March 2021 